\title{
Resistencia de Plasmodium falciparum a tres fármacos antimaláricos en Turbo (Antioquia, Colombia), 1998
}

\author{
Silvia Blair, ${ }^{1}$ Leidy L. Lacharme, ${ }^{1}$ Jaime Carmona Fonseca ${ }^{1}$ \\ y Alberto Tobón ${ }^{1}$
}

RESUMEN En 1998, se determinó in vivo $e$ in vitro la frecuencia y el grado de resistencia de Plasmodium falciparum a los tres fármacos antimaláricos (cloroquina, amodiaquina y sulfadoxina) pirimetamina) más utilizados en el municipio de Turbo (zona de Urabá, Antioquia, Colombia) en una muestra representativa de la población con malaria. Se realizaron análisis clínicos y parasitológicos durante 14 días según la prueba estándar recomendada por la Organización Mundial de la Salud. In vivo, P. falciparum mostró resistencia a la cloroquina, amodiaquina y sulfadoxina/pirimetamina con una frecuencia de 97,7 y 13\%, respectivamente; in vitro, las cifras correspondientes fueron de 21, 23 y 9\%, respectivamente. La concordancia entre los resultados in vivo $e$ in vitro fue de $23 \%$ para la cloroquina.

Palabras clave Antimaláricos, Colombia, farmacorresistencia, malaria/paludismo, Plasmodium falciparum.

En Colombia, el índice parasitario anual (IPA) pasó de 2,3 por mil habitantes en 1967 a 10 por mil en 1998. Antioquia es uno de los departamentos colombianos con mayor incidencia de malaria; en 1967 se registró un IPA de 3,8 por mil y en 1999 la incidencia de malaria por Plasmodium falciparum fue de 32,4 por 100000 habitantes (1). Las zonas antioqueñas de Urabá y el Bajo Cauca fueron consideradas en 1997 como las de mayor riesgo de malaria en el departamento (2).

\footnotetext{
1 Grupo de Malaria, Facultad de Medicina, Universidad de Antioquia. La correspondencia debe ser enviada a Silvia Blair a la siguiente dirección postal: Carrera 51D N ${ }^{\circ} 62-29$, piso 3, Laboratorio de Malaria, Medellín, Colombia. Correo electrónico: sblair@catios.udea.edu.co
}

Se han realizado algunos estudios sobre la resistencia de $P$. falciparum a los fármacos antimaláricos en Colombia (3-5), pero, en general, los datos son escasos. En el mundo, la respuesta a los antimaláricos se ha relacionado, entre otros aspectos, con las diferencias metabólicas individuales (6), los cambios en la absorción gastrointestinal producidos por la dieta o por fármacos (7-9), la participación de cepas de $P$. falciparum poseedoras de genes de resistencia $(10,11)$, la presión farmacológica ejercida por el uso masivo de antimaláricos y las dosis subterapéuticas (12-14).

El presente estudio determinó por métodos in vivo e in vitro la frecuencia y el grado de resistencia de $P$. falciparum a la cloroquina (CQ), amodiaquina (AMO) y sulfadoxina-pirimetamina (SP) en el municipio de Turbo
(Urabá, Colombia), lugar escogido por la alta incidencia de la infección, el uso prolongado de medicamentos antimaláricos y la venta sin receta médica de estos medicamentos.

\section{MATERIALES Y MÉTODOS}

\section{Selección de la muestra}

El cálculo del tamaño de la muestra se hizo a partir del total de casos de malaria por $P$. falciparum $(N=900)$ registrados en Turbo durante el año de estudio (1998) y de la prevalencia de la resistencia in vivo de $P$. falciparum a cada uno de los fármacos según un estudio anterior realizado por nuestro grupo en el municipio de Zaragoza (Antioquia), en el que obtuvimos cifras 
de $67 \%$ para la CQ, 3\% para la AMO y 9\% para la SP. De acuerdo con estos datos, la prevalencia de resistencia (p) en Turbo se estimó en 75\% para la CQ y $10 \%$ para la AMO y la SP. El error de muestreo (d) se fijó en 0,155, se definió un intervalo de confianza de $95 \%$ ( $Z$ = $1,96)$ y un error alfa (nivel de significación) de 0,05. Con estas condiciones, los tamaños de las muestras resultantes fueron de 29 pacientes para la CQ y 14 para la $\mathrm{AMO}$ y la SP, según la siguiente fórmula:

$$
\begin{gathered}
n=N \cdot \mathrm{Z}^{2} \cdot \mathrm{p} \cdot(1-\mathrm{p}) / \\
\left(N \cdot \mathrm{d}^{2}\right)+\left[\mathrm{Z}^{2} \cdot \mathrm{p} \cdot(1-\mathrm{p})\right] .
\end{gathered}
$$

La asignación de los pacientes a cada uno de los tres tratamientos se hizo de forma aleatoria a medida que se iban incluyendo en el estudio. La determinación de la sensibilidad in vitro se hizo con desconocimiento de los resultados in vivo, de tal forma que el investigador que había hecho el seguimiento del paciente no era quien establecía el efecto in vitro.

Los participantes fueron captados en el puesto de diagnóstico de malaria del hospital municipal e informados sobre los objetivos, riesgos y beneficios del estudio; si aceptaban, eran incluidos después de firmar su consentimiento con conocimiento de causa; en el caso de los niños, el consentimiento fue dado por los padres. Los criterios de inclusión fueron los siguientes: 1) edad $>1$ año ; 2) parasitemia mínima de 900 y máxima de 80000 parásitos asexua$\mathrm{dos} / \mathrm{mm}^{3}$ de sangre; 3 ) residencia en el área de estudio, y 4) posibilidad de acudir a las consultas de seguimiento durante 14 días. A su vez, los criterios de exclusión consistieron en: 1) malaria mixta (P. vivax y P. falciparum); 2) malaria grave o complicada; 3) vómitos o diarrea intensos; 4) presencia en orina de 4-aminoquinolinas determinadas mediante la prueba de Bergqvist et al. (15), o de SP o sus metabolitos según la prueba de Mount et al. (16); 5) presencia de enfermedades que requirieran tratamiento o aumentaran el riesgo de reacciones adversas a las sulfonamidas, y 6) antecedentes de hipersensibilidad a los medicamentos utilizados.

\section{Evaluación de la resistencia in vivo}

Se utilizó la prueba estándar de 14 días de la Organización Mundial de la Salud (OMS) para evaluar la resistencia a los fármacos antimaláricos (17). El día cero se comprobaron el diagnóstico de especie y la parasitemia inicial mediante gota gruesa, se realizó un examen clínico y se obtuvo información epidemiológica referente a la edad, sexo, residencia, profilaxis antimalárica, automedicación y uso de antimaláricos en los 20 días anteriores. Se tomaron muestras de sangre para las pruebas de sensibilidad in vitro $\mathrm{y}$ muestras de orina para las pruebas de Mount y Bergqvist. El tratamiento fue administrado personalmente por los investigadores. Las dosis de CQ y AMO fueron de $25 \mathrm{mg} / \mathrm{kg}$ (10 mg/kg el día 0 y 7,5 mg/ $\mathrm{kg}$ los días 1 y 2), y las de SP de 25/1,25 mg/kg (dosis única el día 0). Los pacientes fueron sometidos a exámenes clínicos y parasitológicos los días 1, 2, 3, 7 y 14 del estudio (18).

La respuesta al tratamiento fue evaluada con indicadores parasitológicos y clínicos. La clasificación de la respuesta parasitológica se basó en la densidad parasitaria (DP):

- Resistencia de tipo III (RIII) si la DP del día 2 fue $\geq 100 \%$ de la del día 0 o si la DP del día 3 fue $\geq 25 \%$ de la del día 0.

- Resistencia de tipo II (RII) si la DP del día 3 fue $<25 \%$ de la del día 0 y siguió siendo positiva hasta el día 8 o si fue negativa el día 3, pero se tornó positiva en cualquier momento antes del día 8.

- Resistencia de tipo I (RI) si la DP del día 3 fue negativa, pero la parasitemia reapareció entre los días 8 y 14 o si la DP del día 3 fue $<25 \%$ de la del día 0 y el día 7 fue negativa, pero reapareció después del día 7.

- Sensibilidad/resistencia de tipo I (S/RI) si la DP del día 3 fue negativa o $<25 \%$ de la del día 0 y, además, fue negativa entre los días 4 y 7 .

- Sensibilidad (S) si la DP del día 3 fue negativa y siguió así entre los días 7 y 14, inclusive. Se utilizó este pará- metro (gota gruesa) para la calificación como sensible.

La respuesta clínica al tratamiento fue clasificada como:

- Fracaso precoz del tratamiento si el paciente presentó durante los tres primeros días de seguimiento signos de peligro o de paludismo grave, si la DP del día 2 fue $\geq 100 \%$ de la del día 0 o si la DP del día 3 fue $\geq 25 \%$ de la del día 0 .

- Fracaso tardío del tratamiento si el paciente presentó entre los días 4 y 14 de seguimiento signos de peligro o de paludismo grave con parasitemia, si acudió a una consulta no programada debido a deterioro clínico en presencia de parasitemia o si presentó parasitemia entre los días 7 y 14.

- Respuesta clínica adecuada si el paciente no cumplió ninguno de los criterios anteriores y se confirmó la desaparición del parásito durante el período de seguimiento (19).

Los pacientes fueron retirados del estudio cuando adquirieron enfermedades que necesitaron la administración de otros medicamentos, presentaron reacciones adversas importantes al medicamento administrado o malaria mixta o no acudieron a las citas programadas.

\section{Prueba de sensibilidad in vitro}

La respuesta de $P$. falciparum a la $\mathrm{CQ}, \mathrm{AMO}$ y SP in vitro se determinó mediante la microtécnica establecida por la OMS (20); de acuerdo con los resultados de un trabajo anterior (datos sin publicar) el tiempo de incubación de los parásitos se incrementó a 38 horas para aumentar la maduración de los esquizontes. La prueba se interpretó según las concentraciones que inhiben el 50\% y el 90\% de los parásitos (CI50 y CI90), obtenidas a partir del gráfico logarítmico de la dosis frente a la respuesta. Para considerar que la prueba funcionaba de forma adecuada, en los pozos de control debió obtenerse un crecimiento mínimo del $10 \%$ de esquizontes. 


\section{Análisis estadístico}

La información fue procesada con el programa Epi Info, versión 6.04. Los grupos de tratamiento se conformaron aleatoriamente y mediante una prueba de asociación de $\chi^{2}$ se comprobó la distribución aleatoria de las variables sexo, edad y procedencia, para garantizar la comparabilidad de los grupos según estas variables.

Mediante la prueba de KruskalWallis se comparó la parasitemia inicial entre los tres grupos. También mediante la prueba de Kruskal-Wallis, se compararon los intervalos de parasitemia de las diferentes respuestas parasitológicas (S, S/RI, RI, RII, RIII) con cada tratamiento y en cada día de control. Asimismo, mediante la prueba de la $\chi^{2}$ se evaluó la asociación entre la respuesta parasitológica (S, S/RI, RI, RII, RIII) a cada uno de los tratamientos y las variables sexo, edad (niños y adultos) y residencia. Con la prueba de la $\chi^{2}$ de Fisher se examinó la asociación entre la respuesta al tratamiento in vivo (sensible/resistente) y la respuesta al tratamiento in vitro (sensible/resistente).

\section{RESULTADOS}

\section{Generalidades epidemiológicas}

Ningún paciente se retiró del estudio durante el seguimiento. Los 60 pacientes fueron 22 mujeres (37\%) y 38 hombres $(63 \%)$, con un promedio de 22 años de edad, (3 a 67); 58 (97\%) residían en áreas rurales. El tiempo medio de residencia en la zona fue de 5 años y $66 \%$ de la población tuvo un tiempo de residencia superior a un año. No se encontró asociación estadísticamente significativa $(P>0,05)$ entre el fármaco recibido y cada una de las variables de sexo, edad y procedencia. Veintiséis porciento de los individuos presentaron malaria en los últimos 6 meses y $67 \%$ de ellos tuvieron el último episodio en los dos meses anteriores al estudio; $73 \%$ recibieron tratamientos antimaláricos incompletos y $18 \%$ realizaron profilaxis

CUADRO 1. Parasitemia inicial de los pacientes con Plasmodium falciparum según el tratamiento recibido. Turbo, Antioquia, Colombia, 1998

\begin{tabular}{|c|c|c|c|c|c|c|}
\hline \multirow{2}{*}{$\begin{array}{c}\text { Parasitemia } \\
\left.\text { (parásitos } \times 10^{3} / \mathrm{l}\right)\end{array}$} & \multicolumn{2}{|c|}{$\mathrm{CQ}$} & \multicolumn{2}{|c|}{$\mathrm{AMO}$} & \multicolumn{2}{|c|}{ SP } \\
\hline & No. & $\%$ & No. & $\%$ & No. & $\%$ \\
\hline $0,0-5000$ & 14 & 48,3 & 8 & 53,3 & 12 & 75,0 \\
\hline 5 001-10000 & 8 & 27,6 & 3 & 20,4 & 1 & 6,3 \\
\hline $1000-50000$ & 7 & 24,1 & 4 & 26,6 & 3 & 18,7 \\
\hline Total & 29 & 100 & 15 & 100,3 & 16 & 100 \\
\hline Media & \multicolumn{2}{|c|}{7701} & \multicolumn{2}{|c|}{9690} & \multicolumn{2}{|c|}{5526} \\
\hline Mediana & \multicolumn{2}{|c|}{5737} & \multicolumn{2}{|c|}{2851} & \multicolumn{2}{|c|}{3780} \\
\hline Desviación típica & \multicolumn{2}{|c|}{7443} & \multicolumn{2}{|c|}{11807} & \multicolumn{2}{|c|}{5050} \\
\hline
\end{tabular}

${ }^{a}$ Contraste de medias de la parasitemia inicial (Kruskal-Wallis): $\mathrm{H}=0,509 ; \mathrm{gl}=2 ; \mathrm{P}=0,775391$.

$\mathrm{CQ}$ : cloroquina; AMO: amodiaquina; SP: sulfadoxina/pirimetamina.

antimalárica con fármacos o plantas medicinales; en 56\% había antecedentes de automedicación. En ningún paciente se presentaron efectos adversos imputables a los medicamentos. La distribución porcentual de las variables de sexo, edad, residencia, antecedentes de malaria en los últimos seis meses, tratamientos antimaláricos incompletos y profilaxis antimalárica fue similar en los tres grupos, lo cual refleja la asignación aleatoria de los pacientes y descarta que alguno de los grupos, en particular el tratado con $\mathrm{CQ}$, hubiese quedado con más pacientes con antecedentes de malaria y de exposición previa al fármaco, lo cual podría haber creado un sesgo, por la inclusión de pacientes ya seleccionados como resistentes al fármaco.

\section{Evolución de la parasitemia según el tratamiento}

Setenta y seis porciento de los pacientes tratados con CQ, 73\% con AMO y $81 \%$ con SP presentaron parasitemia < 10000 parásitos $/ \mathrm{mm}^{3}$ al ingresar en el estudio. No hubo diferencia estadísticamente significativa entre los grupos con respecto a los promedios de parásitos en el día 0 (cuadro 1). La evolución de la parasitemia en el período de observación para cada tratamiento y cada respuesta parasitológica se presenta en los cuadros 2,3 y 4 .

\section{Respuesta in vivo según el tratamiento}

Se encontró resistencia in vivo a la CQ en $97 \%$ de los pacientes. El cuadro 2 muestra la distribución según el tipo de resistencia. La parasitemia inicial no presentó una distribución normal. Todos los casos fueron comparables por edad, sexo y zona de procedencia $(P>0,05)$.

Noventa y tres porciento de los pacientes tratados con AMO fueron sensibles y solo un paciente (7\%) presentó S/R-I. La parasitemia inicial no tuvo una distribución normal y su valor medio no presentó diferencias estadísticamente significativas entre los casos sensibles y el caso resistente (cuadro 3). Los pacientes de los grupos sensible y resistente fueron comparables por sexo, edad y zona de residencia $(P>0,05)$.

De los pacientes tratados con SP, $88 \%$ fueron sensibles y $12 \%$ presentaron resistencia de grado II. La parasitemia inicial no presentó una distribución normal; los pacientes sensibles y resistentes fueron comparables por sexo, edad y procedencia (cuadro 4).

\section{Sensibilidad in vitro}

Se lograron cultivar 38 aislados. El control negativo demostró que la prueba in vitro funcionó de forma adecuada y garantizó que los datos 
CUADRO 2. Sensibilidad in vivo a la cloroquina y parasitemia según el día de control en pacientes con Plasmodium falciparum. Turbo, Antioquia, Colombia, 1998

\begin{tabular}{|c|c|c|c|c|c|c|c|}
\hline \multirow[b]{2}{*}{ Respuesta } & \multicolumn{2}{|c|}{ Pacientes } & \multicolumn{5}{|c|}{ Parasitemia media ${ }^{a}$} \\
\hline & No. & $\%$ & Día 0 & Día 2 & Día 3 & Día 7 & Día 14 \\
\hline Sensible & 1 & 3,4 & 908 & 1 & 0 & 0 & 0 \\
\hline Resistente II & 12 & 41,4 & 10536 & 2801 & 745 & 1543 & NA \\
\hline Resistente I & 1 & 3,4 & 3102 & 0 & 2 & 160 & NA \\
\hline Sensible/Resistente I & 2 & 6,9 & 5667 & 637 & 0 & 0 & 6318 \\
\hline
\end{tabular}

CUADRO 3. Sensibilidad in vivo a la amodiaquina y parasitemia según el día de control en pacientes con Plasmodium falciparum. Turbo, Antioquia, Colombia, 1998

\begin{tabular}{|c|c|c|c|c|c|c|c|}
\hline \multirow[b]{2}{*}{ Respuesta } & \multicolumn{2}{|c|}{ Pacientes } & \multicolumn{5}{|c|}{ Parasitemia media $^{a}$} \\
\hline & No. & $\%$ & Día 0 & Día 2 & Día 3 & Día 7 & Día 14 \\
\hline Sensible & 14 & 93,3 & 7697 & 1713 & 174 & 0 & 0 \\
\hline
\end{tabular}

a Prueba de Kruskall-Wallis. No hay diferencia estadísticamente significativa entre las medias del día $0(\mathrm{H}=2,625 ; \mathrm{gl}=1 ; P=0,105193)$.

CUADRO 4. Sensibilidad in vivo a la sulfadoxina/pirimetamina y parasitemia según el día de control en pacientes con Plasmodium falciparum. Turbo, Antioquia, Colombia, 1998

\begin{tabular}{|c|c|c|c|c|c|c|c|}
\hline \multirow[b]{2}{*}{ Respuesta } & \multicolumn{2}{|c|}{ Pacientes } & \multicolumn{5}{|c|}{ Parasitemia media ${ }^{a}$} \\
\hline & No. & $\%$ & Día 0 & Día 2 & Día 3 & Día 7 & Día 14 \\
\hline Sensible & 14 & 87,5 & 5224 & 33 & 11 & 0 & 0 \\
\hline Resistente I & 2 & 12,5 & 7640 & 160 & 450 & 674 & $N A^{b}$ \\
\hline
\end{tabular}

a Prueba de Kruskall-Wallis. No hay diferencia estadísticamente significativa entre las medias del día $0(\mathrm{H}=1,613 ; g \mathrm{l}=1 ; P=0,204008)$.

${ }^{b} \mathrm{NA}$ : no aplicable

obtenidos podían ser usados para evaluar la resistencia. Se encontraron tres casos resistentes a la CQ $(21 \%)$, tres a la $\operatorname{AMO}(23 \%)$ y uno a la SP $(9 \%)$ (cuadro 5). El crecimiento medio de esquizontes mostró una rápida disminución en todos los pozos predosificados. La SP presentó la CI50 en el pozo B (10/0,125 pmol/mL) y la CI90 en el pozo C (30/0,375 pmol/mL); solo un caso presentó crecimiento de esquizontes con más de 8 núcleos con concentraciones $>30 / 0,375 \mathrm{pmol} / \mathrm{mL}$. La concordancia observada entre la resistencia in vivo y la resistencia in vitro para la CQ fue de $23 \%(0+3 / 13)$; un paciente fue sensible in vivo y no se logró cultivar el parásito in vitro (cuadro 6). Con la AMO se presentaron tres casos resistentes in vitro y sensibles in vivo; un paciente fue resistente in vivo y no se consiguió cultivar el parásito in vitro. Con la SP se presentaron dos casos resistentes in vivo y uno resistente in vivo e in vitro; en el otro caso no se pudo cultivar el parásito in vitro.

\section{DISCUSIÓN}

Se encontró in vivo una resistencia de P. falciparum a la CQ de 97\%. Los 60 pacientes con malaria por P. falciparum en quienes se evaluó la respuesta a los tratamientos con CQ, AMO y SP no presentaron diferencias estadísticamente significativas con respecto al sexo, edad y zona de residencia. Es decir, estas variables no pueden explicar las diferencias en la sensibilidad, que tampoco puede explicarse por la parasite- 
CUADRO 5. Sensibilidad in vitro de Plasmodium falciparum a la cloroquina (CQ), amodiaquina (AMO) y sulfapirimetamina (SP). Turbo, Antioquia, Colombia, 1998

\begin{tabular}{lccc}
\hline \multicolumn{1}{c}{ Muestras } & CQ & AMO & SP \\
\hline Sembradas & 29 & 15 & 16 \\
Cultivadas con éxito & 14 & 13 & 11 \\
Sensibles & $11^{\mathrm{a}}$ & $10^{\mathrm{a}}$ & 10 \\
Resistentes & 3 & $3^{\mathrm{b}}$ & 1 \\
\hline
\end{tabular}

a Incluye un caso con respuesta in vivo no conocida y sensible in vitro.

${ }^{b}$ Incluye un caso con respuesta in vivo no conocida y resistente in vitro. mia inicial, porque tampoco presentó diferencias entre los tres grupos.

Las condiciones en las que se realizó la investigación permiten asegurar que los pacientes tomaron los fármacos en el momento adecuado y a la dosis recomendada; los propios investigadores comprobamos que los enfermos ingerían las tabletas y que el fármaco ingerido no se había perdido por vómitos o diarrea, lo que nos impide pensar en una recrudescencia por dosis infraterapéuticas. Para descartar problemas con la calidad de los fármacos, las tabletas de CQ suministradas a los pacientes fueron estudiadas en tres laboratorios diferentes (dos colombianos y uno uruguayo) y se concluyó que contenían la cantidad de CQ base que deben poseer y que tenían la pureza requerida.

Según la OMS, la prueba estándar para evaluar la sensibilidad de $P$. falciparum a la CQ y otros esquizonticidas circulantes es el procedimiento in vivo. La valoración in vivo quizás refleje mejor la respuesta real del enfermo al fármaco, pero puede ser influenciada por variables que quedan fuera de testinal y los cambios en la absorción producidos por la dieta $(8,9)$ y los fármacos (7), o la participación de cepas de $P$. falciparum poseedoras de genes de resistencia $(10,11)$. Pensamos que otros posibles aspectos implicados son las parasitosis gastrointestinales asociadas y la degradación del fármaco en metabolitos sin efecto parasiticida, como la desetilcloroquina y la bidesetilcloroquina. La falta de concordancia entre los estudios in vivo e in vitro es un aspecto aún no definido. control, como la absorción gastroin-
La concordancia observada en este trabajo entre las pruebas in vivo e in vitro para la resistencia a la CQ fue tan solo de $23 \%$.

Testa et al. (21) informaron que no hubo concordancia entre los resultados de las pruebas in vivo e in vitro para medir la resistencia de P. falciparum a la CQ. Por otra parte, en Gambia, Seidlein et al. (22) notificaron resistencia in vitro a la CQ en $72 \%$ de los casos y resistencia in vivo en $17 \%$, mientras que en este trabajo encontramos mayor resistencia in vivo que in vitro. En Burundi (África Central), Coosemans et al. (23) hallaron una resistencia in vivo de $49 \%$ en el día 15 y una resistencia in vitro de $73 \%$, mientras que Adagu et al. (24), en Nigeria, encontraron resistencia in vivo en $16 \%$ de los casos (en el día 28) e in vitro en $38 \%$. Brasseur et al. (25), con el mismo esquema de tratamiento utilizado por nosotros, pero evaluado a los 7 días y con otra técnica para evaluar la resistencia in vitro, hallaron resistencias in vivo de 50 y $52 \%$ en Camerún (ciudad de Bangangté) y en Congo, frente a resistencias in vitro de 58 y $45 \%$, respectivamente. Aunque estos datos muestran más coincidencia entre los resultados obtenidos in vivo e in vitro, no quedan dudas de que la discordancia entre los resultados de estas dos formas de evaluar la resistencia de $P$. falciparum a la CQ es real y de gran magnitud y no es exclusiva de nuestro informe.

La situación del fenómeno de resistencia es muy compleja y solo podemos decir que, con los métodos de rutina actuales, debe aceptarse la existencia de una elevada resistencia de $P$. falciparum a la CQ en el municipio de Turbo. El nivel de resistencia frente al cual se debe dejar de usar un medicamento no está establecido universalmente. En Tailandia se cambiaron los medicamentos de primera línea con una tasa de fracaso parasitológico de 15 a 20\% (resistencia tipo I) y en Malawi se cambió el uso de cloroquina con $75 \%$ de resistencia (19). En Perú se definieron dos umbrales por encima de los cuales se considera que el fracaso de los tratamientos es inadmisible, uno de $12 \%$ y otro de $30 \%$ (19). El Ministerio de Salud de Colombia recomienda que cuando la propor-
CUADRO 6. Concordancia de las respuestas de Plasmodium falciparum a la cloroquina (CQ), amodiaquina (AMO) y sulfapirimetamina (SP) in vivo e in vitro. Turbo, Antioquia, Colombia, 1998

\begin{tabular}{|c|c|c|c|c|c|c|}
\hline \multirow[b]{2}{*}{ Droga } & \multirow{2}{*}{$\begin{array}{c}\text { Respuesta } \\
\text { in vivo }\end{array}$} & \multicolumn{2}{|c|}{$\begin{array}{c}\text { Respuesta } \\
\text { in vitro }\end{array}$} & \multicolumn{2}{|c|}{$\begin{array}{c}\% \\
\text { Resistencia }\end{array}$} & \multirow{2}{*}{$\begin{array}{c}\% \\
\text { Concordancia }^{b}\end{array}$} \\
\hline & & $\mathrm{S}^{\mathrm{a}}$ & $\mathrm{R}^{\mathrm{a}}$ & in vivo & in vitro & \\
\hline \multirow[t]{2}{*}{$C Q$} & S & 0 & 0 & 97 & 21 & 23 \\
\hline & $\mathrm{R}$ & 10 & 3 & 28/29 & $3 / 14$ & \\
\hline \multirow[t]{2}{*}{ AMO } & $S$ & 9 & 2 & 7 & 23 & 82 \\
\hline & $\mathrm{R}$ & 0 & 0 & $1 / 15$ & $3 / 13$ & \\
\hline \multirow[t]{2}{*}{ SP } & S & 10 & 0 & 13 & 9 & 100 \\
\hline & $\mathrm{R}$ & 0 & 1 & $2 / 16$ & $1 / 11$ & \\
\hline
\end{tabular}

a : sensible, R: resistente.

b Solo se refiere a los casos con ambas respuestas conocidas (in vivo e in vitro): 13 en CQ, 11 en AMO y 11 en SPM. La concordancia observada se calcula mediante la suma de resultados sensibles en ambas pruebas más los resistentes en ambas, dividido por el total de resultados. Ejemplo: $C Q(0+3) / 13=23 \%$. 
ción de fracasos terapéuticos alcanza un nivel crítico de $25 \%$ se deben realizar investigaciones con muestras representativas in vivo durante 14 días (26).

En este trabajo la resistencia a la CQ alcanzó el 97\%, lo cual muestra que se han superado los niveles permisibles establecidos por otros países y que, por lo tanto, al menos en Urabá, es necesario plantear una alternativa terapéutica diferente y realizar estudios de evaluación de la farmacorresistencia en el país.

Los resultados obtenidos en esta investigación, sumados a los que recientemente se han logrado en Zaragoza (Antioquia) (datos sin publicar) y en Quibdó (Chocó) (5), ponen de manifiesto un acelerado crecimiento de la resistencia de $P$. falciparum a la cloroquina en Colombia, fenómeno paralelo al incremento de la resistencia a otros medicamentos, de otras especies de Plasmodium y en otros lugares del mundo. Esta situación nos lleva a plantear la necesidad de que todos los países se comprometan a fondo con la aplicación inmediata de las estrategias de lucha antimalárica definidas en Amsterdam en 1992 (27), las cuales hacen énfasis en el empleo selectivo de medidas preventivas, siempre que puedan llevar a resultados sostenibles técnica y financieramente, en la participación comunitaria y en la coordinación del trabajo sanitario con los otros sectores (agricultura, educación, medio ambiente, entre otros). Esto incluye, por supuesto, la educación masiva, intensificada y prolongada de la población y de los agentes de salud para evitar el mal uso de los medicamentos antimaláricos, así como la creación y ejecución de un sistema de vigilancia centinela de la resistencia a los fármacos.

Agradecimientos. Agradecemos a la Dirección Seccional de Salud de Antioquia por la cofinanciación del proyecto, a la Universidad de Antioquia por el apoyo académico y financiero, a las directivas y empleados del hospital "Francisco Valderrama" de Turbo (Urabá) y a Leonardo A. Ríos, Sofía Martínez y Elizabeth Díaz, integrantes del Grupo de Malaria de la Universidad de Antioquia.

\section{REFERENCIAS}

1. Estadísticas de malaria por P. vivax y P. falciparum en Antioquia 1994-1999. Dirección Seccional de Salud de Antioquia. Disponible en: http://www.gobant.gov.co/dssa/malaria.htm

2. Dirección Seccional de Salud de Antioquia. Bol Epid Ant 1997. Julio, Agosto, Septiembre, 22:309-311.

3. Moore DV, Lanier SE. Observations of two Plasmodium falciparum infections with an abnormal response to chloroquine. Am J Trop Med Hyg 1961;10:5-9.

4. Restrepo A, Alvarez L, Restrepo M. Estudio in vivo de la resistencia del $P$. falciparum a la cloroquina en Colombia. Acta Med Col 1980;5: 367-379.

5. Osorio L, Giraldo L, Grajales L, Barat L, Córdoba F, Arriaga A, et al. Evaluación in vivo de la resistencia de Plasmodium falciparum a cloroquina y sulfa/pirimetamina en Quibdó, Chocó. Biomédica 1997;17(supl 2):201-202.

6. Longworth DL. Drug-resistant malaria in children and in travelers. Pediatr Clin North Am 1995;42:649-664

7. McElnay JC, Mukhtar HA, D'Arcy PF, Temple DJ. In vitro experiments on chloroquine and pyrimethamine absorption in the presence of antacid constituents or kaolin. J Trop Med Hyg 1982;85:153-158.

8. Tulpule A, Krishnaswamy K. Effect of food on bioavailability of chloroquine. Eur J Clin Pharmacol 1982;23:271-273.

9. Tulpule A, Krishnaswamy K. Effect of rice diet on chloroquine bioavailability. Drug Nutr Interact 1983;2:83-86.

10. Cox-Singh J, Singh B, Alias A, Abdullah MS. Assessment of the association between three pfmdr1 point mutations and chloroquine re- sistance in vitro of malaysian Plasmodium falciparum isolates. Trans R Soc Trop Med Hyg 1995;89:436-437.

11. Povoa MM, Adagu IS, Oliveira SG, Machado RL, Miles MA, Warhurst DC. Pfmdr1 Asn1042Asp and Asp1246Tyr polymorphisms, thought to be associated with chloroquine resistance, are present in chloroquineresistant and -sensitive Brazilian field isolates of P. falciparum. Exp Parasitol 1998; 88:64-68.

12. Mockenhaupt FP. Mefloquine resistance in Plasmodium falciparum. Parasitol Today 1995;1: 248-253.

13. World Health Organization. Vector bionomics and the epidemiology and control of malaria. Part III. Document CTD/MAL/96.1, 1996.

14. Di Perri G, Olliaro P, Nardi S, Deganello R, Allegranzi B, Bonora S, et al. Response of uncomplicated falciparum malaria to oral chloroquine and quinine in Burundi highlands. Acta Trop 1998;70:25-33.

15. Bergqvist $Y$, Hed C, Funding L, Suther A. Determination of chloroquine and its metabolites in urine: a field method based on ion-pair extraction. Bull World Health Organ 1985;63: 893-898.

16. Mount DL, Green MD, Zucker JR, Were JB, Todd GD. Field detection of sulfonamides in urine: the development of a new and sensitive test. Am J Trop Med Hyg 1996;55:250-253.

17. Organización Mundial de la Salud. Políticas sobre medicamentos antimaláricos: necesidades de información, tratamiento de la malaria no complicada y manejo de la malaria en el embarazo. WHO/MAL/94.1070. 1994.

18. Colombia, Ministerio de Salud. Dirección General de Promoción y Prevención. Guía clínica para el diagnóstico y tratamiento de la malaria en Colombia. Santa Fe de Bogotá: Ministerio de Salud; 1998.

19. Perú, Ministerio de Salud. Resistencia de Plasmodium falciparum a medicamentos antimaláricos en el Perú, Proyecto Vigía, Informe Técnico. Lima: Ministerio de Salud; 1999.

20. World Health Organization. In vitro test (mark II) for the assessment of the response of Plasmodium falciparum to chloroquine, mefloquine, quinine, sulfadoxine/pyrimethamine and amodiaquine. Document MAP/87.2 revision $1,1990$.

21. Testa J, Traore LK, Nabalma S, Sondo B, Guissou IP. Chloroquine resistance of Plasmodium falciparum. Study of a surveillance method based on placental apposition and determination of blood chloroquine in pregnant women. Sante 1998;8:293-296.

22. Von Seidlein L, Duraisingh MT, Drakeley CJ, Bailey R, Greenwood BM, Pinder M. Polymorphism of the Pfmdr1 gene and chloroquine resistance in Plasmodium falciparum in the Gambia. Trans R Soc Trop Med Hyg 1997;91:450-453.

23. Coosemans $\mathrm{MH}$, Hendrix L, Barutwanayo $\mathrm{M}$, Butoyi G, Onori E. Resistance medicamenteuse de Plasmodium falciparum au Burundi (Afrique Centrale). World Health Organization. WHO/MAL/84.1014. 1984.

24. Adagu IS, Warhurst DC, Ogala WN, AbduAguye I, Audu LI, Bamgbola FO, et al. Antimalarial drug response of Plasmodium falciparum from Zaria, Nigeria. Trans R Soc Trop Med Hyg 1995;89:422-425.

25. Brasseur P, Agnamey P, Ekobo AS, Samba G, Favennec L, Kouamouo J. Sensitivity of Plas- 
modium falciparum to amodiaquine and chloroquine in central Africa: a comparative study in vivo and in vitro. Trans R Soc Trop Med Hyg 1995;89:528-530.

26. Colombia, Ministerio de Salud: Subdirección de Ambiente y Salud. Diagnóstico y trata- miento de la malaria en Colombia. Santa Fé de Bogotá: Ministerio de Salud; 1999.

27. OMS. Conferencia Ministerial sobre paludismo. Estrategia Mundial de la Lucha contra el Paludismo. Documento CTD/MCM/92.3. p 29.
Manuscrito recibido el 14 de junio de 2000 y aceptado para publicación, tras revisión, el 5 de diciembre de 2000

ABSTRACT In 1998 we determined in vivo and in vitro the frequency and the degree of resistance of Plasmodium falciparum to the three antimalarials (chloroquine, amodiaquine, and sulfadoxine/pyrimethamine) most utilized in the municipality of Turbo (in the area of Urabá, Antioquia, Colombia), in a sample representative of the population with malaria. We carried out clinical and parasitological analyses over a 14-day period using the standard test recommended by the World Health Organization. In vivo, $P$. falciparum showed resistance to chloroquine, amodiaquine, and sulfadoxine/ pyrimethamine, with a frequency of $97 \%, 7 \%$, and $13 \%$, respectively. In vitro, the corresponding figures were $21 \%, 23 \%$, and $9 \%$, respectively. For chloroquine the level of agreement between the in vivo and in vitro results was $23 \%$.

\section{Resistance of Plasmodium falciparum to three antimalarials in Turbo (Antioquia, Colombia), 1998}

Vaccine Research: international conference

\author{
Dates: $\quad$ 23-25 April 2001 \\ Location: Hyatt Regency Crystal City Hotel \\ Arlington, Virginia, United States of America
}

The Fourth Annual Conference on Vaccine Research will serve as a major international noncommercial scientific forum devoted exclusively to the presentation and sharing of data and knowledge among the diverse disciplines of vaccinology.

There will be presentations and discussions on the latest vaccine-related scientific data, results, and issues. Among the specific topics to be discussed at the meeting are: the economics of vaccines; ethical aspects of vaccinology; vaccination, autoimmunity, and autoimmune diseases; vaccines against malignancies and chronic diseases; control of meningococcal disease by vaccination; new approaches to vaccine development; and issues in vaccine safety.

The conference is intended for researchers and scientists, epidemiologists, microbiologists, immunologists, molecular biologists, public health officials, physicians, veterinarians, and vaccine manufacturers.

The official language for the conference is English.

The conference is sponsored by the National Foundation for Infectious Diseases, in collaboration with the Centers for Disease Control and Prevention, the National Institute of Allergy and Infectious Diseases, the International Society for Vaccines, the U.S. Department of Agriculture, the Center for Biologics Evaluation and Research, the Albert B. Sabin Vaccine Institute, and the World Health Organization.

The registration fee is $\$ 325$ if paid by 23 March and $\$ 375$ after that date. The fee does not cover the cost of hotel accommodations.

Information:

National Foundation for Infectious Diseases

Suite 750, 4733 Bethesda Ave.

Bethesda, Maryland 20814-5228, United States of America

Telephone: (301) 656-0003, ext. 19

Fax: (301) 907-0878

E-mailinfo@nfid.org

World Wide Web site: http://www.nfid.org/conferences/vaccine01/ 